\title{
Optimal and Robust Saturated Control for a Clock Generator
}

\author{
C. Albea S. Lesecq and D. Puschini
}

\begin{abstract}
Fine-grain Dynamic Voltage and Frequency Scaling (DVFS) is becoming a requirement for Globally-Asynchronous Locally-Synchronous (GALS) architectures. However, the area overhead of adding voltage and frequency control engines in each voltage/frequency island must be taken into account to optimize the circuit. This paper focuses on the control for the frequency actuator. An optimal and robust saturated control law, with a minimum hardware implementation area is proposed for a Clock Generator, taking into account the delay introduced by the sensor. This controller is designed with LyapunovKrasovskii theory that ensures asymptotic stability, disturbance rejection as well as system robustness with respect to delay presence and parameter uncertainties. The closed-loop system presents a regional stabilization due to the actuator saturation. An estimation of a maximum attraction domain is provided. The performance achieved with this controller are shown in simulation.
\end{abstract}

\section{INTRODUCTION}

The continuous increase in clock frequency together with technology scaling has made the distribution of a single global clock over a digital chip tremendously difficult. Globally Asynchronous Locally Synchronous (GALS) design alleviates the problem of clock distribution by having multiple clocks, each one being distributed on a small area of the chip. An architecture with different clock frequency domains appears as a natural enabler for fine-grain power-aware architectures. Actually, power consumption is a limiting factor in Very Large Scale Integration (VLSI), especially for mobile applications. Voltage-Frequency Islands (VFI) [1] have proven to be highly effective to reduce the power consumption of the chip while meeting the performance requirements [2]. The key idea behind local DVFS is to control at fine grain the supply voltage and the frequency of an island at runtime to minimize its power consumption of the island considered while satisfying the computation/throughput constraints [3].

DVFS techniques mainly rely on two 'actuators'. These actuators need to be dynamically controlled in order to reduce the power consumption while maintaining the required performance. More precisely, the control policy

This work has been conducted while C. Albea was with CEA LETI-MINATEC Campus, Grenoble, France. Now, C.Albea is with both CNRS; LAAS; 7 avenue du colonel Roche, F-31077 Toulouse, France and University of Toulouse; UPS, INSA, INP, ISAE; LAAS; F-31077 Toulouse, France. carolina.albea@gmail.com

S. Lesecq and D. Puschini are with CEA LETI-MINATEC Campus, 17 rue des Martyrs, 38000, Grenoble, France \{suzanne.lesecq, diego.puschini\}@cea.fr must be carefully designed in order to achieve high power efficiency at low area cost. The voltage actuator fixes the supply voltage of the VFI. It can be a classical buck converter [4] or a discrete Vdd-hopping converter [5], [6]. The frequency actuator is supposed to be a Clock Generator.

An important issue of technology scaling is the in-die and die-to-die process variability (P-variability). As a consequence, each VFI performance and optimal functional frequency will strongly differ from place to place within the chip. These changes are also induced by Voltage and Temperature variations (VT-variability) over time. Therefore, the optimum functional and energetic point of the whole circuit can be found if VFI number $i$ has its functioning frequency in the range $\left[F_{\min , i}, F_{\max , i}\right]$ [7]. If the clock is generated for the whole circuit, and distributed in each VFI, the maximum acceptable frequency (i.e. that will ensure no timing fault for any VFI) will be $F_{\max , i}=\min \left\{F_{\max , i}, \quad \forall i\right\}$, leading to a suboptimal circuit functioning. Therefore, the clock is locally generated and controlled by a Clock Generator according to Process, Voltage and Temperature (PVT) variations.

The main objective of this paper is to design a control law for the Clock Generator (see Fig.1) taking into account the next objectives:

- robustness with respect to PVT variations;

- low cost implementation;

- saturation;

- delay consideration and

- exogenous disturbance rejection.

Therefore, the designed controller must not only guarantee the set-point stabilization, but also other criterions.

An optimal and robust saturated control law for the Clock Generator is proposed in discrete time. The system is rewritten into a suitable state-space representation to formulate a robust $H_{\infty}$ control problem that can be solved using Lyapunov-Krasovskii theory [8]. In this process, the saturation in the actuator input is considered [9]. The designed controller guarantees asymptotic stability, disturbance rejection as well as robustness of the system with respect to delays and uncertain parameters. The problem is expressed in terms of Linear Matrix Inequalities (LMIs). Likewise, an attraction domain is estimated in such a way that a regional stabilization for the saturated control is guaranteed. The robustness properties of the closed-loop system are confirmed by simulations. 
The rest of this paper is organized as follows: in Section II, the error equation of the circuit model is derived as well as its properties. The robustness problem statements are presented in Section III. Likewise, in Section IV, the stabilization of the time-delay system is performed. This result is extended for a robust control design in Section V. An optimization procedure is proposed in Section VI. The control gains are computed in Section VII, being confirmed by simulations in Section VIII. The paper closes with a section of conclusion.

Notation. For a given $x \in \mathcal{R}$,

$$
\text { sat }_{m}^{M}(x) \triangleq\left\{\begin{array}{cll}
M & \text { if } & x>M \\
x & \text { if } & m \leq x \leq M \\
m & \text { if } & x<m .
\end{array}\right.
$$

and $\operatorname{round}(x)$ is the nearest integer to $x$. For a given set $\mathcal{S}, \mathcal{C} o(\mathcal{S})$ denotes the convex hull of set $\mathcal{S} . \Delta \zeta \triangleq \zeta^{+}-\zeta^{-}$, where $\zeta^{+}$and $\zeta^{-}$are respectively $\zeta(k+1)$ and $\zeta(k)$, i.e., the value of $\zeta$ in two consecutive sampling times. Finally, $\mathcal{L}_{2}$ is the space of $\left\{x_{k}\right\}$ with $\left\|x_{k}\right\|_{2}^{2} \triangleq \sum_{k=0}^{\infty} x_{k}^{T} x_{k}<\infty$.

\section{Error equation of the Clock Generator}

The main blocks of the Clock Generator considered in this paper are a digital to analog converter (DAC), a Voltage Controlled Oscillator (VCO) that provides at its output a frequency $f(t)$, a sensor to measure this frequency and a controller that contains a frequency comparator between the targeted frequency, $f_{r}$, and the output frequency and some 'intelligent controller'. Note that this structure is similar to the one of a Digital Frequency-Locked Loop. Figure 1 shows a sketch of the Clock Generator.

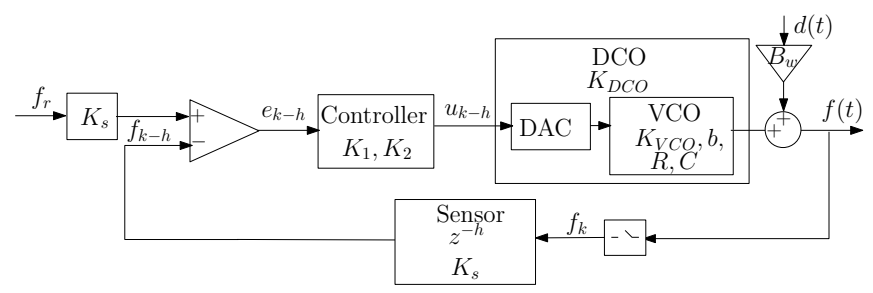

Fig. 1: Clock Generator block diagram.

The main blocks of this Clock Generator are modeled through design considerations and accurate simulations. The VCO can be modeled as a RC circuit [10]. Indeed, it can be approximately modeled as

$\dot{f}(t)=-\frac{f(t)}{R C}+K_{V C O} u(t-\tau)+b+B_{w} d(t), \quad \tau=h\left(t_{k+1}-t_{k}\right)$

where $f \in \mathcal{R}^{1}$ is the analog frequency output, $u$ is a control signal, $d$ is a $\mathcal{L}_{2}$ disturbance and $K_{V C O}$ and $B_{w}$ are constant. $R$ and $C$ represent the resistive and capacitive components, respectively, of the circuit, and $b$ is an offset.

This model is approximately discretized, in such a way that the whole Digitally-Controlled Oscillator (DCO) is modeled by :

$$
f_{k+1}=\left(1-\rho T_{s}\right) f_{k}+K_{D C O} T_{s} u_{k-h}+b T_{s}+B_{w} T_{s} d_{k}
$$

where $T_{s}$ is the sampling period, $K_{D C O}$ is the gain of the whole DCO and $\rho \triangleq \frac{1}{R C}$.

Defining $e_{k} \triangleq f_{r}-f_{k}$, where $f_{r}$ is the reference signal, the error equation is

$e_{k+1}=\left(1-\rho T_{s}\right) e_{k}-K_{D C O} T_{s} u_{k-h}+\rho T_{s} f_{r}-b T_{s}-B_{w} T_{s} d_{k}$

A controller proposed for this system is given by

$$
u_{k-h}=\operatorname{sat}_{\bar{u}_{m}}^{\bar{u}_{M}}\left\{u_{k-h-1}+K x_{k-h}\right\},
$$

where $h$ is the size of the constant delay introduced by the sensor, $x_{k-h} \triangleq\left[\begin{array}{ll}e_{k-h} & e_{k-h-1}\end{array}\right]^{T}$ and $K \triangleq\left[\begin{array}{ll}K_{1} & K_{2}\end{array}\right]$. Note that the controller proposed presents a simple enough structure to be implemented in hardware. This property is very important in SoCs [11].

\section{Problem statement}

The problem statement is formulated. The closed-loop system (1) is rewritten in a state-space form taking into account the delay, saturation, uncertain parameters and any exogenous disturbance.

Parameters $R, C, T_{s}$ and $K_{D C O}$ can be considered uncertain. Their corresponding uncertainty intervals are

- $R \in\left[R^{m}, R^{M}\right]$,

- $C \in\left[C^{m}, C^{M}\right]$,

- $T_{s} \in\left[T_{s}^{m}, T_{s}^{M}\right]$,

- $K_{D C O} \in\left[K_{D C O}^{m}, K_{D C O}^{M}\right]$.

Consequently, the main objective is to find the optimal gain $K$, in such a way that, Control (2) is robust with respect to delays as well as parameter uncertainties. Likewise, this optimal $K$ must guarantee asymptotic stability and disturbance rejection for the known constant delay $h$.

A. Alternative representation for the saturated control (2) and the error equation (1)

Firstly, some lemmas are given to rewrite the saturated control (2) in an alternative form.

Define $\chi \triangleq\left[\begin{array}{c}u_{k-1-h_{1}} \\ x_{k-h}\end{array}\right]$. Note, from Eq.(1), $u_{k-1-h_{1}}$ directly depends on $x_{k}=\left[e_{k}, e_{k-1}\right]$.

Lemma 1: [12], let $K, G \in \mathbb{R}^{1 \times 2}$ be given. For all, $\chi_{k-h} \in \mathbb{R}^{1 \times 3}$, if $\chi_{k-h} \in\left\{\chi_{k-h} \in \mathbb{R}^{1 \times 3}:\left[\begin{array}{ll}1 & G\end{array}\right] \chi_{k-h} \in\right.$ $\left.\left[\begin{array}{ll}1 & N\end{array}\right]\right\}$, then

$$
\operatorname{sat}_{1}^{N}\left\{\left[\begin{array}{ll}
1 & G
\end{array}\right] \chi_{k-h}\right\} \in \mathcal{C} o\left\{\left[\begin{array}{ll}
1 & K
\end{array}\right] \chi_{k-h}, \quad\left[\begin{array}{ll}
1 & G
\end{array}\right] \chi_{k-h}\right\} .
$$

Lemma 2: Assume that there exists $G \in \mathbb{R}^{1 \times 2}, c>0$ and $\Psi \triangleq \operatorname{diag}\left\{\rho, P_{1}\right\}$, where $P_{1}^{\dagger}>0 \in \mathbb{R}^{2}, \rho>0 \in \mathbb{R}^{1}$ such that for any $\chi_{k-h} \in \mathfrak{X}$, where

$$
\mathfrak{X}=\left\{\chi_{k-h}: \chi_{k-h}^{T} \Psi \chi_{k-h} \leq c^{-1}\right\},
$$

\footnotetext{
${ }^{\dagger} P_{1}$ is a positive matrix defined to guarantee system stability.
} 
then, $1<u_{k-1-h_{1}}+G x_{k-h}<N$, and Control (2) admits the following representation

$$
\begin{aligned}
u_{k-h_{1}}= & {\left[\alpha_{k}\left(u_{k-1-h_{1}}+K x_{k-h}\right)\right.} \\
& \left.+\left(1-\alpha_{k}\right)\left(u_{k-1-h_{1}}+G x_{k-h}\right)\right] \\
= & {\left.\left[u_{k-1-h_{1}}+\alpha_{k} K x_{k-h}+\left(1-\alpha_{k}\right) G x_{k-h}\right)\right] } \\
= & {\left[u_{k-1-h_{1}}+\bar{u}_{k-h}\right], }
\end{aligned}
$$

where $\bar{u}_{k-h} \triangleq\left(\alpha_{k} K+\left(1-\alpha_{k}\right) G\right) x_{k-h}$ with $\alpha_{k} \in[0,1]$, for all $k>0$.

Then, Eq. (1) can be rewritten as

$$
\begin{aligned}
e_{k+1} & =\left(1-\rho T_{s}\right) e_{k}-b T_{s}+\rho T_{s} f_{r}-B w T_{s} d_{k} \\
& -K_{D C O} T_{s}\left(u_{k-1-h_{1}}+\bar{u}_{k-h}\right)
\end{aligned}
$$

\section{B. State-space representation}

The saturated control law and error equation, as redefined above, allow system (1) rewrite it in a state-space form. From Eq. (1),

$$
u_{k-h}=\frac{-e_{k+1}+\left(1-\rho T_{s}\right) e_{k}+\rho T_{s} f_{r}-b T_{s}-B_{w} T_{s} d_{k}}{K_{D C O} T_{s}},
$$

and, therefore

$$
u_{k-h-1}=\frac{-e_{k}+\left(1-\rho T_{s}\right) e_{k-1}+\rho T_{s} f_{r}-b T_{s}-B_{w} T_{s} d_{k-1}}{K_{D C O} T_{s}}
$$

which substituted in (4) gives

$$
\begin{aligned}
e_{k+1} & =\left(2-\rho T_{s}\right) e_{k}+\left(\rho T_{s}-1\right) e_{k-1}-K_{D C O} T_{s} \bar{u}_{k-h} \\
& +T_{s} B_{w}\left(d_{k}-d_{k-1}\right)
\end{aligned}
$$

From Lemma 1 and 2, Eq. (5) can be rewritten in the following matrix form:

$$
x_{k+1}=A x_{k}+B \bar{u}_{k-h}+\bar{B}_{w} w_{k}
$$

where $w_{k} \triangleq\left[\begin{array}{ll}d_{k} & d_{k-1}\end{array}\right]^{T}$,

$$
\begin{gathered}
A \triangleq\left[\begin{array}{cc}
2-\rho T_{s} & \rho T_{s}-1 \\
1 & 0
\end{array}\right], \quad B \triangleq\left[\begin{array}{c}
-K_{D C O} T_{s} \\
0
\end{array}\right], \\
\bar{B}_{w} \triangleq\left[\begin{array}{cc}
T_{s} b_{w} & -T_{s} b_{w} \\
0 & 0
\end{array}\right] .
\end{gathered}
$$

\section{Stability and disturbance rejection problem}

Equation (6) can be rewritten in the following explicit closed-loop form, in such a way that a $H_{\infty}$ problem can be formulated.

$$
\begin{aligned}
x_{k+1} & =A x_{k}+B\left(\alpha_{k} K+\left(1-\alpha_{k}\right) G\right) x_{k-h}+\bar{B}_{w} w_{k} \\
x_{l} & =\phi_{l}, \quad \forall l \in[-h, 0] \\
z_{k} & =I_{2} x_{k}
\end{aligned}
$$

where $x_{k}, z_{k}, w_{k} \in \mathcal{R}^{2}$ are the state vector, controlled output and exogenous disturbance input, respectively. $\phi_{l}$ is the initial condition and $h \geq 0 \in \mathcal{R}$ is a fixed and known delay.

Problem 1: The problem is to find $\mathfrak{X}\left(P_{1}, c\right)$, vector $G$ and $K$, such that

a) Lemma 2 holds and, hence, the closed-loop system (7), and b) there exists a Lyapunov-Krasovskii functional $V_{k}>$ 0 such that $V_{k+1}-V_{k}$ along the solution of (7) fulfills

$$
V_{k+1}-V_{k}<0
$$

when the system is not perturbed, and for any disturbance input, there exists a minimum disturbance attenuation, $\gamma^{*} \geq 0$, such that, for all $\gamma \geq \gamma^{*}$ the $\mathcal{L}_{2}$ gain between the disturbance vector $w_{k}$ and the output vector $z_{k}$ is less or equal to $\gamma$. i.e.

$$
\begin{aligned}
& \left\|z_{k}\right\|_{2}^{2}-\gamma^{2}\left\|w_{k}\right\|_{2}^{2}<0, \quad \forall w_{k} \in \mathcal{L}_{2} \\
& \text { for } \quad \phi_{l}=0, \quad-h \leq l \leq 0 .
\end{aligned}
$$

The solution to this problem guarantees the asymptotic convergence, system stability as well as the disturbance rejection for the time-delay system (7)-(9).

\section{IV. $H_{\infty}$ CONTROL DESIGN}

In order to cope with Problem 1 a mathematical manipulation of Eq. (7) is performed via a descriptor model transformation [13]. The descriptor approach is a variable change, which makes easier to work with Lyapunov-Krasovskii functional [8].

\section{A. Descriptor model transformation}

Equation (7) is manipulated in order to achieve the previous objectives. A descriptor model transformation is applied.

Considering $y_{k} \triangleq x_{k+1}-x_{k}, \quad \psi_{k} \triangleq \sum_{i=k-h}^{k-1} y_{i}$, Eq. (7) is rewritten in the descriptor form [13]:

$$
\begin{aligned}
& {\left[\begin{array}{c}
x_{k+1} \\
0
\end{array}\right]=} \\
& {\left[\begin{array}{c}
y_{k}+x_{k} \\
-y_{k}+A x_{k}-x_{k}+B\left(\alpha_{k} K+\left(1-\alpha_{k}\right) G\right) x_{k-h}+\bar{B}_{w} w_{k} .
\end{array}\right]}
\end{aligned}
$$

From $x_{k-h}=x_{k}-\psi_{k}$, this system can be compactly written as:

$$
E \bar{x}_{k+1}=\bar{A} \bar{x}_{k}-\left[\begin{array}{c}
0 \\
B\left(\alpha_{k} K+\left(1-\alpha_{k}\right) G\right)
\end{array}\right] \psi_{k}+\left[\begin{array}{c}
0 \\
\bar{B}_{w}
\end{array}\right] w_{k},
$$

where

$$
\begin{aligned}
& \bar{A} \triangleq\left[\begin{array}{cc}
I_{2} & I_{2} \\
A+B\left(\alpha_{k} K+\left(1-\alpha_{k}\right) G\right)-I_{2} & -I_{2}
\end{array}\right] \\
& E \triangleq \operatorname{diag}\left\{I_{2}, 0_{2}\right\}, \quad \bar{x}_{k} \triangleq\left[\begin{array}{l}
x_{k} \\
y_{k}
\end{array}\right] .
\end{aligned}
$$

\section{B. Condition for state-space representation}

Condition a) of Problem 1 is satisfied, if

$$
1<u_{k-1-h_{1}}+G x_{k-h}<N, \quad \forall \chi_{k-h} \in \mathfrak{X}
$$

given in (3) is guaranteed.

Subtracting $\frac{N+1}{2}$ in inequality (13) and from [14], it is seen that, it is necessary satisfy

$$
\begin{aligned}
2 N-2 & >N\left(1+c x_{k-h}^{T} P_{1} x_{k-h}+c u_{k-1-h}^{T} \rho u_{k-1-h}\right)-2 \\
& >4 u_{k-1-h}+4 G x_{k-h}-2(N+1)
\end{aligned}
$$


From Lemma 2, relationships (14) correspond to

$$
\left[\begin{array}{c}
1 \\
u_{k-1-h_{1}} \\
x_{k-h}^{T}
\end{array}\right]^{T}\left[\begin{array}{ccc}
3 N & -2 & -2 G \\
-2 & c N \rho & 0 \\
-2 G^{T} & 0 & c N P_{1}
\end{array}\right]\left[\begin{array}{c}
1 \\
u_{k-1-h_{1}} \\
x_{k-h}
\end{array}\right]>0
$$

This inequality is satisfied if

$$
\left[\begin{array}{ccc}
c & -2 & -2 Y \\
-2 & \rho 3 N^{2} & 0 \\
-2 Y^{T} & 0 & 3 N^{2} \bar{P}_{1}
\end{array}\right]>0
$$

Note that this LMI is equivalent to (15) by means of employing the Schur's complement, defining $Y \triangleq G Q_{1}$ with $Q_{1} \in \mathbb{R}^{2}$ which is Hermitian, applying $\bar{P}_{1}=Q_{1} P_{1} Q_{1}$ and pre- and post-multiplying by $\operatorname{diag}\left\{1,1, Q_{1}\right\}$.

\section{Control design}

Now, condition b) of Problem 1 can be formulated in terms of Linear Matrix Inequalities (LMIs) [8]. Fulfillment of conditions (10) and (11) is looked for.

Assumption 1: There exists a Lyapunov function $V_{k}$, with condition (10) and a $\gamma$, such that,

$$
V_{k+1}-V_{k}+z_{k+1}^{T} z_{k+1}-\gamma^{2}\left(w_{k}^{T} w_{k}\right) \leq \zeta^{T} \Gamma \zeta<0 .
$$

where $\zeta \triangleq\left[\begin{array}{lll}\bar{x}_{k} & \psi_{k} & w_{k}\end{array}\right]^{T}$ is an augmented state vector and $\Gamma \in \mathcal{R}^{4 \times 4}$ is a symmetric matrix.

Define $P \triangleq\left[\begin{array}{cc}P_{1} & P_{2} \\ P_{2} & 0\end{array}\right]$, being $P_{2}$ Hermitian.

The function $\Gamma$ is obtained defining the next Lyapunov-Krasovskii candidate

$$
V_{k}=V_{1, k}+V_{2, k}+V_{3, k}
$$

being

$$
\begin{aligned}
V_{1, k} & =\bar{x}_{k}^{T} E P E \bar{x}_{k} \\
V_{2, k} & =\sum_{n=1}^{h} \sum_{i=k-n}^{k-1} y_{i}^{T} R y_{i}, \quad R>0 \\
V_{3, k} & =\sum_{i=k-h}^{k-1} x_{i}^{T} S x_{i}, \quad S>0,
\end{aligned}
$$

where $V_{1, k}$ guarantees asymptotic stability of system (12) without delays. Delay-dependent as well as delayindependent criteria are considered in $V_{2, k}$ and $V_{3, k}$, respectively [8], [15].

Next, a sufficient condition for asymptotic stability and disturbance rejection is derived.

Theorem 1: Consider system (7)-(9) with energybounded $w_{k}$ and control law $\bar{u}_{k-h}=\alpha_{k} K x_{k-h}+(1-$ $\left.\alpha_{k}\right) G x_{k-h}$ where $h>0 \in \mathcal{R}$ is a known constant delay and $K, G \in \mathcal{R}^{1 \times 2}$. If there exist $S, R \in \mathcal{R}^{2 \times 2}$ such that

$$
\begin{aligned}
& P_{1}>0, \\
& \Gamma_{k}<0 \\
& \text { LMIs }
\end{aligned}
$$

where $\Gamma_{k}$ is defined in (24) found at the top of next page, then the equilibrium of the closed-loop system (7)-(9) is asymptotically stable and there is a value $\gamma^{*}$ such that for $\gamma<\gamma^{*}$ condition (11) is fulfilled.

Proof: The goal is to satisfy $V_{k+1}-V_{k}+z_{k}^{T} z_{k}-$ $\gamma^{2} w_{k}^{T} w_{k}<0$ for both disturbance rejection and asymptotic stability of the equilibrium for system (12).

Lyapunov-Krasovskii method yields:

$$
\begin{aligned}
& V_{1, k+1}-V_{1, k}=\bar{x}_{k+1}^{T} E P E \bar{x}_{k+1}-\bar{x}_{k}^{T} E P E \bar{x}_{k} \\
& =\left\{\bar{x}_{k}^{T} \bar{A}^{T}-\psi_{k}^{T}\left[\begin{array}{ll}
0 & \alpha_{k} K^{T} B^{T}+\left(1-\alpha_{k}\right) G^{T} B^{T}
\end{array}\right]+\right. \\
& \left.+w_{k}\left[\begin{array}{ll}
0 & \bar{B}_{w}^{T}
\end{array}\right]\right\} P\left\{\bar{A} \bar{x}_{k}-\left[\begin{array}{c}
0 \\
\alpha_{k} B K+\left(1-\alpha_{k}\right) B G
\end{array}\right] \psi_{k}\right. \\
& \left.+\left[\begin{array}{c}
0 \\
\bar{B}_{w}
\end{array}\right] w_{k}\right\}-\bar{x}_{k}^{T} E P E \bar{x}_{k}=\bar{x}_{k}^{T}\left[\bar{A}^{T} P \bar{A}-E P E\right] \bar{x}_{k} \\
& -\bar{x}_{k}^{T} \bar{A}^{T} P\left[\begin{array}{c}
0 \\
\alpha_{k} B K+\left(1-\alpha_{k}\right) B G
\end{array}\right] \psi_{k} \\
& -\psi_{k}^{T}\left[0 \quad \alpha_{k} K^{T} B^{T}+\left(1-\alpha_{k}\right) G^{T} B^{T}\right] P \bar{A} \bar{x}_{k} \\
& -\bar{x}_{k}^{T} \bar{A}^{T} P\left[\begin{array}{c}
0 \\
\bar{B}_{w}
\end{array}\right] w_{k}+w_{k}\left[\begin{array}{ll}
0 & \bar{B}_{w}^{T}
\end{array}\right] P \bar{A} \bar{x}_{k} .
\end{aligned}
$$

From (20) and Jensen Inequality [16]:

$$
\begin{aligned}
V_{2, k+1}-V_{2, k} & =h y_{k}^{T} R y_{k}-\sum_{n=1}^{h} y_{k-n}^{T} R y_{k-n} \\
& \leq \bar{x}_{k}^{T}\left[\begin{array}{cc}
0 & 0 \\
0 & h R
\end{array}\right] \bar{x}_{k}-\frac{1}{h} \psi_{k}^{T} R \psi_{k}
\end{aligned}
$$

Finally,

$$
\begin{aligned}
V_{3, k+1}-V_{3, k} & =x_{k}^{T} S x_{k}-x_{k-h}^{T} S x_{k-h} \\
& =x_{k}^{T} S \psi_{k}+\psi_{k}^{T} S x_{k}-\psi_{k}^{T} S \psi_{k}
\end{aligned}
$$

These developed expressions are applied to inequality (17), in such a way that the LMIs (23) are obtained.

\section{RoBust CONTROL}

The uncertain parameters given in Section III are now taken into account, at the same time that stability as well as disturbance rejection for the time-delay Vddhopping system are ensured. For this reason, Theorem 1 is extended in the case of polytopic uncertainties.

Denote $\Omega \triangleq\left[\begin{array}{lll}A & B & \alpha_{k}\end{array}\right]$ and assume that $\Omega \in$ $\mathcal{C} o\left\{\Omega_{j}, \quad j=1, \ldots, 16\right\}$, namely

$$
\Omega=\sum_{j=1}^{n} \lambda_{j} \Omega_{j}, \quad 0 \leq \lambda_{j} \leq 1, \quad \sum_{j=1}^{n} \lambda_{j}=1
$$

and being the vertices of the polytope described by $\Omega_{j}=$ $\left[A^{(j)} \quad B^{(j)} \quad \alpha_{k}^{(j)}\right]$ for $j=1, \ldots, 16$.

Pre- and post-multiplying LMI (23) by $Q=$ $\operatorname{diag}\left\{Q_{1}, Q_{1}, Q_{1}, I_{2}\right\}$ and taking $Q_{1}=P_{2}^{-1}>0$ and $\bar{P}_{1}=Q_{1} P_{1} Q_{1}, \bar{R}=Q_{1} R Q_{1}, \bar{S}=Q_{1} S Q_{1}$, the following sufficient condition is achieved.

Theorem 2: Consider system (7)-(9) with energybounded $w_{k}$ and control law $\bar{u}_{k-h}=\alpha_{k} K x_{k-h}+(1-$ $\left.\alpha_{k}\right) G x_{k-h}$ for $\alpha_{k} \in[0,1]$, where $h \geq 0 \in \mathcal{R}$ is a known constant delay and $K, G \in \mathcal{R}^{1 \times n}$. If there exist $T, Y \in$ 


$$
\Gamma_{k} \triangleq\left[\begin{array}{ccc}
\bar{A}^{T} P \bar{A}-E P E+\operatorname{diag}\left\{I_{2}, h R\right\} & -\bar{A}^{T} P\left[\begin{array}{c}
0 \\
B \alpha_{k} K+\left(1-\alpha_{k}\right) G
\end{array}\right]+\left[\begin{array}{c}
S \\
0
\end{array}\right] & \bar{A}^{T} P\left[\begin{array}{c}
0 \\
\bar{B}_{w}
\end{array}\right] \\
* & -\frac{1}{h} R-S & 0 \\
* & * & -\gamma^{2} I_{2}
\end{array}\right]
$$

$\mathcal{R}^{n \times 1}$ and $Q_{1} \in \mathcal{R}^{n \times n}$ with $K=T Q_{1}^{-1}, G=Y Q_{1}^{-1}$ and $\bar{R}, \bar{S}>0 \in \mathcal{R}^{n \times n}$ for $j=1, \ldots, 16$ such that

$$
\begin{aligned}
& \bar{P}_{1}>0 \\
& \bar{\Gamma}^{(j)}<0 \quad j=1, \ldots ., 16,
\end{aligned}
$$

LMIs (16)

being $\bar{\Gamma}^{(j)}$ defined in (27) found at the top of next page, are satisfied. Then, in the vertices $i$ and $j$, the equilibrium is asymptotically stable as well as the disturbances are rejected in the entire polytope.

Proof: This is an extension of Theorem 1 for polytopic uncertainties with some mathematical manipulations. Therefore, this theorem proof follows Theorem 1 proof.

Remark 1: This robust control tuning method is conservative due to the definition of matrix $P$, as well as, the attraction domain, $\mathfrak{X}$.

Corollary 1: Gain $K$, obtained from $T$ and $Q_{1}$ in Theorem 2, fulfills Theorem 1 and consequently guarantees both robust stability and robust disturbance rejection for a fixed delay.

\section{Optimal And Robust CONTROL}

In order to obtain a maximum attraction domain, i.e. minimum $c$, and for the disturbances to be effectively rejected (i.e. minimum $\gamma$ ), an optimal control problem is formulated.

Assumption 2: There exists a functional cost

$$
J \triangleq x_{k+1} P_{1} x_{k+1}+\left\|z_{k+1}\right\|_{2}^{2}-\gamma^{2}\left\|w_{k}\right\|_{2}^{2}
$$

The first term on the right side quantifies the size of the attraction domain and the other terms the disturbance attenuation.

Lemma 3: Suppose that Assumptions 1 and 2 are fulfilled, Then the optimal controller gain $K$ for Problem 1 can be found by:

Minimize $-J$

subject to:

$$
\begin{aligned}
& \bar{\Gamma}^{(j)}<0 \\
& \bar{P}_{1}>0 . \\
& \text { LMIs }^{(j)}(16) \quad j=1, \ldots, 16 .
\end{aligned}
$$

Proof: The optimal Problem 1 is solved by Lemma 1 if condition (11) is fulfilled [17].

For $w_{k} \neq 0$ and under zero initial conditions

$$
V_{k+1}-V_{k} \leq-z_{k+1}^{T} z_{k+1}+\gamma^{2} w_{k}^{T} w_{k}
$$

The summation of both side is

$$
V_{k+1}-V_{0} \leq-\sum_{k=0}^{k+1} z_{k+1}^{T} z_{k+1}+\gamma^{2} \sum_{k=0}^{k} w_{k}^{T} w_{k}
$$

For $k \rightarrow \infty$, under the zero initial condition $V_{0}=0$ and the positive definitiveness of the Lyapunov function, it is proved that

$$
\begin{aligned}
& \sum_{k=0}^{\infty} z_{k+1}^{T} z_{k+1} \leq \gamma^{2} \sum_{k=0}^{\infty} w_{k}^{T} w_{k} \Rightarrow \\
& \left\|z_{k+1}\right\|_{2}^{2} \leq \gamma^{2}\left\|w_{k}\right\|_{2}^{2} .
\end{aligned}
$$

Corollary 2: The optimal gain $K$, obtained applying Lemma 3 guarantees both robust stability and robust disturbance rejection and provides a maximum attraction domain.

\section{Optimal And Robust CONTROL RESUlt}

In this section, the robust control gains for Control (2) are computed. For the control signal $\bar{u}_{m}=0.6 \mathrm{~V}$ and $\bar{u}_{M}=1.1 \mathrm{~V}, b=-26 \mathrm{MHz}$ and $h=1 . b_{w}=10$ was taken, for any exogenous disturbance. The uncertain parameters take the following ranges:

- $C \in[2, \quad 4] n F$,

- $R \in[5,10] \Omega$,

- $K_{D C O} \in[10,1000] M H z / L S B$,

- $T_{s} \in[20,30] n s$.

From Lemma 3, it comes that

$$
K_{1}=0.8 \quad K_{2}=-0.8
$$

Likewise $\gamma=0.025, c=0.9, G=\left[\begin{array}{ll}0.0747 & -0.0732\end{array}\right]$ and $P_{1}=\left[\begin{array}{cc}0.0062 & -0.0062 \\ -0.0062 & 0.0062\end{array}\right]$.

Note that even if the control constant tuning is conservative, there is a feasible solution.

\section{Simulation Results.}

In this section, some simulations show the robustness of the optimal and robust saturated control law proposed for the Clock Generator. For these simulations, the data given above are taken.

Figure 2 shows some simulation results when the system parameters are changed:

syst 1: $K_{D C O}=0.04 \frac{G H z}{L S B}, R=5 \Omega, C=2 \cdot 10^{-9} F, T_{s}=20 \mathrm{~ns}$,

syst 2: $K_{D C O}=0.14 \frac{G H z}{L_{S} B}, R=5 \Omega, C=2 \cdot 10^{-9} F, T_{s}=20 \mathrm{~ns}$,

syst 3: $K_{D C O}=0.04 \frac{G H z}{L S B}, R=10 \Omega, C=410^{-9} F, T_{s}=20 \mathrm{~ns}$,

syst 4: $K_{D C O}=0.04 \frac{G H z}{L S B}, R=5 \Omega, C=2 \cdot 10^{-9} F, T_{s}=30 n s$.

'syst 1', 'syst 3' and 'syst 4' are superposed. Note that the four systems converge to the set-point, showing the system robustness.

Finally, note in Fig. 3 that the control law rejects an added exogenous disturbance, as expected. 


$$
\bar{\Gamma}^{(j)} \triangleq\left[\begin{array}{cccc}
\bar{\Gamma}_{1}^{(j)} & \bar{\Gamma}_{2}^{(j)} & -\alpha_{k}^{(j)} B^{(j)} T-\left(1-\alpha_{k}^{(j)}\right) B^{(j)} Y+\bar{S} & \bar{B}_{w} Q_{1} \\
* & \bar{P}_{1}-2 Q_{1}+h \bar{R} & -\alpha_{k}^{(j)} B^{(j)} T-\left(1-\alpha_{k}^{(j)}\right) B^{(j)} Y & \bar{B}_{w} Q_{1} \\
* & * & -\frac{\bar{R}}{h}-\bar{S} & 0 \\
* & * & * & -\gamma^{2} Q_{1}
\end{array}\right], \quad j=1, \ldots, 16 .
$$

where

$$
\begin{aligned}
& \bar{\Gamma}_{1}^{(j)} \triangleq Q_{1} A^{(j)^{T}}+A^{(j)} Q_{1}-2 Q_{1}+\alpha_{k}^{(j)} T^{T} B^{(j)^{T}}+\left(1-\alpha_{k}^{(j)}\right) Y^{T} B^{(j)^{T}}+\alpha B_{k}^{(j)} T+\left(1-\alpha_{k}^{(j)}\right) B^{(j)} Y+I_{2} I_{2}^{T} \\
& \bar{\Gamma}_{2}^{(j)} \triangleq \bar{P}_{1}+Q_{1} A^{(j)^{T}}-2 Q_{1}+\alpha_{k}^{(j)} T^{T} B^{(j)^{T}}+\left(1-\alpha_{k}^{(j)}\right) Y^{T} B^{(j)^{T}},
\end{aligned}
$$

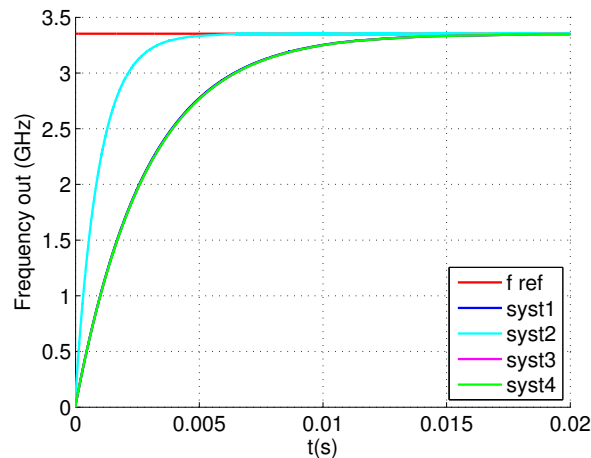

Fig. 2: Evolution of the output frequency for four different systems.

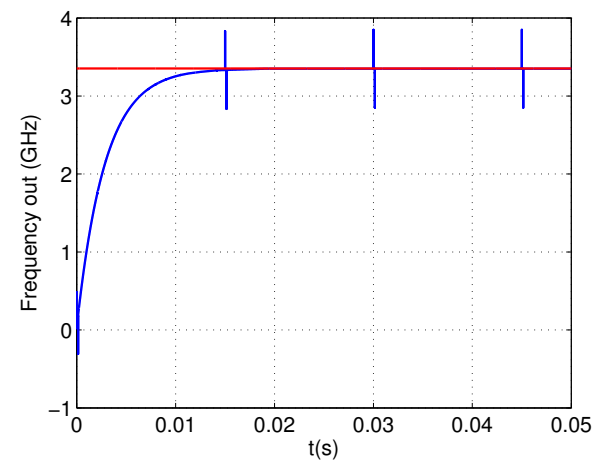

Fig. 3: Exogenous disturbance added to 'syst 1'.

\section{COnClusions}

In this paper an optimal and robust saturated controller was designed for a time-delay Clock Generator. The system is rewritten in a suited state-space representation, such that an optimal and robust $H_{\infty}$ problem can be formulated to tune the control gains. This problem is dealt with Lyapunov-Krasovskii theory, which provides some stability conditions through Linear Matrix Inequalities (LMIs). Consequently, robust equilibrium stability as well as robust disturbance rejection under parameter uncertainties are ensured for the time-delay system. The method also takes into account the control saturation estimating a maximum attraction domain. The closed-loop system robustness is exemplified by means of simulations.

\section{REFERENCES}

[1] P. Choudhary and D. Marculescu, "Hardware based frequency/voltage control of voltage frequency island systems," in In Proc. IEEE Hardware/Software Codesign and System Synthesis (CODES+ISSS), 2007, pp. 34-39.

[2] M. Horowitz, T. Indermaur, and R. González, "Low-power digital design," in IEEE Symposium Low Power Electronics, 2002 , pp. 8-11.

[3] K. Choi and M. Soma, R. Pedram, "Fine-grained dynamic voltage and frequency scaling for precise energy and performance tradeoff based on the ratio of off-chip access to on-chip computation times," IEEE Trans on Computer-Aided Design of Integrated Circuits and Systems, vol. 24, pp. 18-28, 2004.

[4] P. Liu, J. Liu, and L. Geng, "A dynamic buck converter with ultra fast response and low voltage ripples designed for DVS systems," IEICE Electronics Express, vol. 6, no. 21, pp. 1490$1496,2009$.

[5] C. Albea, C. Canudas-de Wit, and F. Gordillo, "Control and stability analysis for the Vdd-hopping mechanism," In Proc. IEEE Conference on Control Applications (CCA), 2009.

[6] S. Miermont and M. Vivet, P. Renaudin, "A power supply selector for energy-and area-efficient local dynamic voltage scaling," Integrated Circuit and System Design. Power and Timing Modeling, Optimization and Simulation, pp. 556-565, 2007.

[7] C. Tretz, C. Guo, and L. Jacobowitz, "An all digital frequencylocked loop immune to hysteresis effects for power management of multicore processors," in Proc. IEEE ProncSOI Conference (SOI), 2010, pp. 1-2.

[8] E. Fridman and U. Shaked, "Delay-dependent $H_{\text {inf }}$ control of uncertain discrete delay systems," European Journal of Control, vol. 11, no. 1, pp. 29-39, 2005.

[9] Y. Cao, Z. Lin, and T. Hu, "Stability analysis of linear timedelay systems subject to input saturation," IEEE Trans. on Circuits and Systems I, vol. 49, no. 2, pp. 233-240, 2002.

[10] H. Bui and Y. Savaria, "Design of a high-speed differential frequency-to-voltage converter and its application in a $5-\mathrm{GHz}$ frequency-locked loop," IEEE Trans on Circuits and Systems I: Regular Papers, vol. 55, no. 3, pp. 766-774, 2008.

[11] G. Micheli, "Nanosystems," Nanosystems Design and Technology, pp. 1-21, 2009.

[12] T. Hu and Z. Lin, Control systems with actuator saturation: analysis and design. Birkhauser, 2001.

[13] E. Fridman and U. Shaked, "A Descriptor System Approach to $H_{\infty}$ Control of Linear Time-Delay Systems," IEEE Trans. on Automatic Control, vol. 47, no. 2, pp. 253-270, 2002.

[14] E. Fridman, A. Pila, and U. Shaked, "Regional stabilization and $H_{\infty}$ control of time-delay systems with saturating actuators," International Journal of Robust and Nonlinear Control, vol. 13, no. 9, pp. 885-907, 2003.

[15] K. Gu and S. Niculescu, "Stability Analysis of Time-Delay Systems: A Lyapunov Approach," Advanced Topics in Control Systems Theory, Lecture Notes from FAP, pp. 139-170, 2005.

[16] K. Gu, V. Kharitonov, and J. Chen, Stability of time-delay systems. Birkhauser, 2003.

[17] P. Millan, L. Orihuela, C. Vivas, and F. Rubio, "An optimal control $L_{2}$-gain disturbance rejection design for networked control systems," in In Proc. IEEE American Control Conference (ACC), 2010, pp. 1344-1349. 